\title{
Classification Of Covid19 Using Deep Neural Network
}

\author{
K.Thaiyalnayaki ${ }^{1}$ \\ \{thaiyalk@ srmist.edu.in ${ }^{1}$ \} \\ Department of Electronics and Communication Engineering, \\ SRM Institute of science and Technology, Ramapuram
}

\begin{abstract}
The ongoing logical researches are applying AI and machine learning in the field of clinical pneumonia and Covid in X-ray beam arrangement. A Deep CNN based model can distinguish the COVID-19 cases at a quicker speed by identifying the features of infected patients. The trainset consists of chest xrayof 80 healthy people and 80 covid people. The test set include 20 normal people and 20 covid affected chest xrays.Keras sequential Deep CNN classifier is constructed with convolution1,pooling1, convolution2,pooling2, flattening and dense fully connected net.The input shape of $2 \mathrm{D}$ convolution layer is of size $64 \times 64 \times 3$ with a $3 \times 3$ mask and ReLu activation function. Max pooling mask size is $2 \times 2$.Conolution layer2 and max pool layer2 utilize the mask $3 \times 3$ and 2x2.After straightening, the completely connected layer comprises of 128 neurons with Relu activation function and one sigmoid neuron.Binary cross entropy loss function and Adam optimizer are used in CNN. The fully connected output layer consists of 129 neurons, out of which 128 are relu activation with 8,02,944 trainable parameters with accuracy obtained is $97 \%$, which can be improved by increasing the dataset.This model act as an automatic classification of covid 19 and normal subjects.
\end{abstract}

Keywords: COVID 19, Deep CNN, X ray, automatic classifier.

\section{Introduction}

The novel Covid in the wake of take up through the respiratory tract of human, influences the lungs of the individual basically, give rise to positions like and extreme than the familiar Pneumonia. The lungs begin to be loaded up with liquid, get aroused and create patches called ground Glass Opacity. Since a great deal of exertion are happening for uncovering the compelling prescription of Covid-19 however upto now the main successful path for security is social removing and lockdown of different urban areas in the nation. Along these lines, it is obligatory to set up a wellbeing center framework dependent on Artificial Intelligence which identify the cases quick and precisely to forestall this regular pandemic. Xbeam machines give reasonable and quicker outcomes to examining of different vital internal organs in the medical clinics. The understanding of different X-beams pictures is normally accomplished physically by specialist radiologists. As information researcher, in the event that it is trained by those caught images with the criticalness of profound discovering that will be an extraordinary guide to clinical specialists for recognizing the COVID-19 sufferers. This will succour creating things in which the X-beam facility is accessible yet the accessibility of a specialist is as yet a fantasy. In measure of scope, it is expected to build up a deep neural 
network (DNN)that can dissect the X-beam pictures of lungs and identify if the individual tests positive for the infection or not. Convolutional Neural Networks (CNN) have proved to be extremely successful in PC vision and clinical picture examination function, amidst other deep learning classifiers. The aftereffects of CNN have demonstrated its cogency in planning of picture information to an exact and anticipated yield. Since the lungs are the essential objective of the infection, dissecting their progressions can give an unequivocal aftereffect of quality of the infection. A CNN based model, can prepare the pictures of Covid tainted lungs and such types of sound lungs. The model will differentiate COVID-19 situations more rapidly by defining the highlights of infected patients in X-ray images of the lungs as dim or shadowy patches. [1].

Traditional classification of image can be partitioned into three principle stages: picture pre processing, include extraction and highlight order. Another work used a ConvNet model trained from scratch on datasets to group and indicate the presence of pneumonia in a group of chest X-ray image samples. The training accuracy achieved is of order $95.31 \%$.Some even introduced an early conclusion concept based on Xception and VGG16 from Pneumonia chest X-beam images. Kermany et al provided an information base containing almost 5000 frontal chest X-beam pictures in the Kermany X-beam data collection, 1500 normal cases, 4100 strange pneumonia occurrences in this investigation. The preliminary results indicated that VGG-16 organization superior than X-ception network with a grouping pace of $87 \%$. Xception network is more apt for grouping X-beam pictures than VGG-16 organization. Varshni et al. suggested highlight extractors (VGG-16, Xception, Res50, Dense-121, and Dense) to be used in conjunction with various classifiers such as SVM, Random Forest, k-nearest neighbours, and Nave Bayes to determine the location of normal and abnormal pneumonia. [2]

To recognize the Pneumonia instances from chest X-beams, a viable CNN calculation is registered with the 121-layered convolutional neuralnetwork. The framework is prepared on an informational collection restrining more than 10000 images giving frontal perspective on lung X-beam and depicts 14 sorts of infirmities given by Rajpurkar et al. Additionally, in the present situation of COVID-19, a CNN based methodology has been pertained to the Xbeam pictures of the chest, since it makes comparative indications Pneumonia. Alqudah et al. have suggested a mechanised COVID-19 technique. They used AI classifiers such as the Support Vector Machine (SVM), irregular backwoods, K-Nearest Neighbor (KNN), and CNN with soft-max to aid in identification with a $98 \%$ accuracy rate. [3]

To assist clinical doctors, compressed sensing (CS) with a deep exchange learning model was used to programme the order of pneumonia on X-beam images. The dataset used in this study consisted of approximately 5800 X-beam data from Kaggle, divided into two classes: abnormal and typical. According to extensive simulation findings, the recommended solution correctly classifies pneumonia as unusual/ordinary with just 2.66 percent misclassification.

\section{Deep Convolutional Neural Network (DNN)}

Deep learning strategies are used to discover the explanatory index features, also incl uding images and videos, that were hidden in the original informationset. The framework mod el is focused on the application of deep learning based CNN. It comprises of 20 layers. The main layer displays the information layer and has a fixed size of $256 \times 256 \times 3$ pixe ls,rendering it an RGB image. The following 19 layers are the mix of 2D Convolution, activation ReLU and MaxPooling layers.Such layers are essential for the VGG16 Models 
pretrained

situationand were created using the ImageNet dataset.VGG16 had the choice of achieving 92. 7 percent exactness on ImageNet, which includes about 15 million explained images from 22, 100 different categories.[4] Along these lines, VGG16 model is utilized for including extraction as a base model. At that point an transfer learning model is applied utilizing the 6 unique layers and created the conceptual model on the COVID-19 tuple. The principal layer is an Average Pooling 2D layer with a pool size of $4 \times 4$, and these 6 layers are a fundamental feature of the imaging system. The standard estimate of the apparent multitude of pixels in the cluster is chosen in standard pooling, but the maximum value is chosen in maxpooling.

A flatten layer whose task is to smooth the tensor changing over the twodimensional lattice ofhighlights into a vector that can be handled by a fully associated classificationalgorith $\mathrm{m}$.After changing thevector, it contributes to a fully deep associatedlayer with a dimension of 64 components and a ReLU enactment feature.Dropout is appliedwith 0.4 limit at

that stage. Taking into account the limit value, the dropout layereffectively ignores a fewneuro ns units. Finally, a yield layer with just two units. The Adam analyser[5] is usedtostreamline t he model. Adaptable moment estimation, also known as Adam, is a timesaving approximation that replaces the standard stochastic gradient drop.DNNs can remove insight from a dataset, resulting insuperhuman displays in a multitude of scenarios. Furthermore, effective DNN design combination has recentlybeen investigated. These models are not only accurate in forecasting, but they are also computationallyeffective. As a result, for COVID-19, analysts chose to use DNNs as AI analysis estimates.

To build such a classifier, several works combine preprepared Convolution Neural Network(CNN)design with transfer learning on the chest xray dataset .As a result,experts started collecting CT and chest Xray image datasets and making them publicly available.Analyzed are deeplearning methodologies for determining and recognising COVID 19 in included patients.Two AIbased strategies for characterization and finding of patients andtypical individ uals lung images, are presented to address the conclusion problem. The primarystrategy is bas ed on ANN and

fractaltechniques for arrangement and highlight extraction. The methodology's exactness and c ognizance were dissected in the second phase usingconventional CNN techniques

\subsection{Deep convolutional neural network Layers}

Convolutional neural networks (CNN) are a form of deep learning technique in which a few layers are efficiently instructed. To reduce preprocessing, CNN employs a variety of $\mathrm{m}$ ultilayered perspectives. A CNN is made up of three basic layers: the convolutional layer, thep ooling layer, the flatten information layer, and the fully associated layer, with each layerperfor ming a different role. There aretwo phases of preparation in any design: forward andback propagation. The input moves from the hidden layer to the yield layer in the forwardprogressio $\mathrm{n}$ and the data simply moves forward.The input would be sent to the hidden layer by the information layer and then output is generated. When a neural organisation is characterised the collected image is passed onto the network in the main stage by backpropagation. The estimation of the network error is calculated when the output is reachedt his value is then given to the network alongside the cost work graph to refresh the network weights.CNN has several types of hidden sublayers, which are mentioned below. The convolut ion layer is at the core of the convolution network, and its output is a threedimensional heap of 
neurons.In simple terms, this layer's output is a threedimensional heap. The CNN network, lik e the featuremaps, uses various parts to convolve the input images in these layers.

The algorithm for classifying the covid -19 with a chest X-ray using DNN is given below. 1.Input data: Chest X-ray Images of Healthy and Patients.

2: Output data: COVID-19 Chest X-ray image is detected as the transfer model .

3: Steps for Pre-processing

4: The X-ray input is resized to 512 x 512 pixels in height and width.

5: Show X-ray images

6: Every X-ray data input should be mean normalised.

7: $\mathrm{Z}=$ Alexnet, Googlenet, Resnet18 download and reuse transfer models

8: The last layer of each model is replaced by transfer model with $4 \times 1$ layer dimension.

9:The output value is given between 1 and -1 by hyperbolic tangent function .

10:Throughout the subtracting sampling task, Max and Average pooling, accumulating in the $\mathrm{CNN}$ is used to outline the feature.

11:Convolutional neural network $(\mathrm{CNN})$ conceptual architecture and training for the division of tainted tissue in pulmonary images.

Confusion matrix, in which evaluation standards are analyzed in a grid is a network used to portray the presentation of a classification model on a lot of trial information for which the genuine values are known. The outcomes shows the loss worth and precision as a component of preparing emphases. The plots are portrayed while preparing the network and the cycle was ended subsequent to acquiring the best outcomes with higher precision and lower network loss for 300 iterationsThe last prepared model was evaluated on both the train and test sets. The model accurately predicts 190 cases out of 204 instances in the test set, according to the confusion matrices of these forecasts. As a result, the test set's precision is 93.1 percent. The model achieves a false negative rate of $2 \%$ for COVID-19 positive cases, demonstrating its adequacy in raising alerts for COVID-19 cases. Other assessment metrics for this order function include a $2 \%$ miss rate and a 9.4 percent result on the test sample. This number is significantly higher than DNN's potential for discovery. CNN, on the other han $\mathrm{d}$, is a leader in the grouping of images due to its higher accuracy. Different aspects are also beneficial to CNN techniques. Drop out for DNN strategies, for example, indicates the strategylevel of misdiagnosis. This importance is almost unheard of when it comes to CNN estimates. However, it isn't insignificant in terms of DNN techniques. As a result of the discov ery, the provided CNN calculation high potential can be used to analyse COVID19 patients' X -Rays.

The

true positive rate versus false positive rate is plotted on receiver operating characteristic (RO C) curve. For each image in the segmentation calculation, this basis is exceptional.

Regardless of whether plots with a higher true positive rate and a lower false positive rateprov e that the image cycle is superior, the results show that the most extreme number of ROC bend is a basically high proficiency. For understanding the ROC bend with solid qualities, zone under the bend is considered ,AUC esteem. This model whether to get esteems near one, shows superior preprocessed ground truth picture for the out put layer. For preparing information, $70 \%$ of images were utilized and the remainder of the images utilized for approval of the introduced design. 


\section{Deep CNN Architecture}

Figure 1 depicts the Deep CNN architecture. The constructed work, Deep CNN mode 1 has 7 layers and requires a $64 \times 64 \times 3$ input image. In all framework, the underlying learning ra te is 2e-4.The model is picked on account of its capacity of simple streamlining, combines quicker, and improvement in the precision with expanded profundity .However, time utilization is enlarged with an expansion in layers of each network. At that point the convolution layer makes a component guide to foresee the class probabilities for each element map got. The part of the main convolution layer, i.e., conv1 is to give low-level highlights like shading, edges, and inclination activity, and so forth. The more deeper convolution layer gives significant level highlights in Xray pictures and also restricted in the more deeper layer.The down sampled spatial size of convolution features are utilized. There are three types of pooling layers: maximum pooling, normal pooling, and global pooling.

The pooling layer acquires the most important features, such as positional invariant a nd rotational one.The completely connected layer gets the pooling layer's straightened output a nd acts as a feedforwardnetwork. The softmax layer keeps the yield in the $[0,1]$ range. As a re sultthe softmax layer forecasts the probability of the knowledge vector in a scolarly class. The number of yields is equal to the number of parallel classes in the chest xray image dataset used to construct the model. The number of outputs in the proposed work would be two.
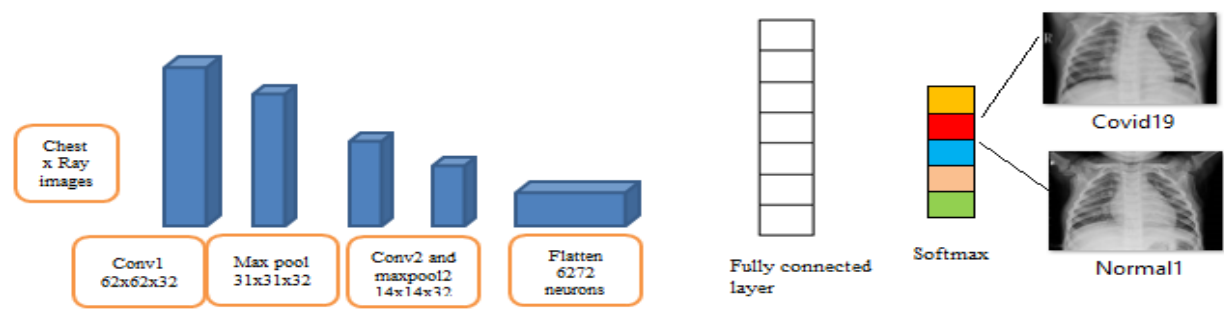

Fig.1 Architecture of Deep CNN

The architecture uses a skip association to include the output from a previous layer in to a subsequent layer, ensuring that data is not corrupted from the primary layer to a deeper la yer. In CNN models, theRectified Linear Unit (ReLU) is a type of enactment function.TheCN Nbased models are actualized by skipping the twofold or triple layer with nonlinearities (ReL $\mathrm{U})$ and using an extra weight structure to familiarise themselves with the weights. This aids de ep learning groups in resolving the vanishing gradient problem. The remaining

network gradually reestablishes the skipped layers as it learns the component space. The featur es map is generated using the first convolutional layer (conv1) and

the deeper layer from the preprepared transfer learning model. The first convolutional layer is use d to test low levelfeatures such as texture, colour, and edges (conv 1).The output activation is obtained byp 
assing the testing image (a positive chest Xray image from Covid19) through the bestaccompli shing network. Furthermore, all activations are scaled to a range of [0 1], where ' 0 ' represents $t$ he lowest activation and ' 1 ' represents the highest activation. Conv2 and the pooling layer are $t$ wo of the model'sdeeper layers. Feature maps reflect the highlights learned by themodel on the chest Xray datasets used in these layers. Furthermore, the most grounded activation channel is used to obtain the features essential for irregularity limitation in COVID-

19 positive chest Xrays.

\section{Experimental Results}

The dataset is downloaded from kaggle for healthy people chest xray and covid19 affected chest xray. The trainset consists of 80 healthy people and 80 normal people chest xray. The test set include 20 normal people and 20 covid affected chest xrays.Keras sequential Deep CNN classifier is constructed as convolution1,pooling1, convolution2,pooling2, flattening and dense fully connected net.The input shape of 2D convolution layer is of size $64 \times 64 \times 3$ with a $3 \times 3$ mask and ReLu activation function.Max pooling mask size is $2 \times 2$.Conolution layer 2 and max pool layer 2 utilize the mask $3 \times 3$ and $2 \times 2$.After straightening, the completely connected layer comprises of 128 neurons with Relu activation function and one sigmoid neuron.Binary cross entropy loss function and Adam optimizer are used in CNN.As there are less number of covid samples, image augmentation is implemented with rescale $=1 . / 255$,shear_range $=0.2$,zoom_range $=0.2$ with horizontal_flip.For a binary classifier , 64x64 targetsize and batchsize of 32 is chosen.Test set is chosen as validation data and the classifier is run for 25 epochs.

The accuracy obtained is $97 \%$ and the corresponding validation accuracy is 97.5\%.The total trainable parameters are 813,217 . With training of 8 lakh parameters in CNN ,the time taken is comparably less. The convolution layer 1 consists of $62 \times 62$ with 32 filter outputs.Max pooling 1 consists of $31 \times 31 \times 32$ and the second convolution layer output size is $29 \times 29 \times 32$.The max pooling layer2 is $14 \times 14$ with 32 filters. Once the $2 \mathrm{D}$ data is converted to single dimension there are 6272 neurons. The fully connected output layer consists of 129 neurons, out of which 128 are relu activation with 8,02,944 trainable parameters as given in table 1 .

Table 1. Sequential classifier model parameters

\begin{tabular}{lll}
\hline No. of Layer & Output size & Parameters \\
\hline 2D Convolution & $62,62,32$ & 896 \\
2D Maxpooling & $31,31,32$ & 0 \\
Conv2D-2 & $29,29,32$ & 9248 \\
2D Maxpooling-2 & $14,14,32$ & 0 \\
Flatten & 6272 & 0 \\
Dense & 128 & 802,944 \\
Dense 1 & 1 & 129 \\
\hline
\end{tabular}

\section{Results and Discussion}

The training and validation accuracy are plotted with the number of epochs, The training accuracy reaches $97 \%$. The number of training set and test need to be increased to increase the training accuracy. The training and validation loss is shown in figure 2 and 3.This network can also be used to help treat patients by separating the infected areafrom their 
lung images. This discovery can also be used to test and manage patients that aredeveloping ta inted regions. There are various works that use artificial intelligence consciousness to divide a nd classify patients, such as diagnosing brain tumours, illness, and cellular cancer in the lungs. Nonetheless, the implementation of these methodologies nearly is quiet. These methodologies nearly utilized in a wearable monitoring framework for conclusion of infection, checking, and moving to specific doctors. Coronavirus pandemic shows that science has a drawn out future point of view. The time has come to execute artificial intelligence reasoning techniques in medication to help specialist's conclusion better and at the earliest opportunity.

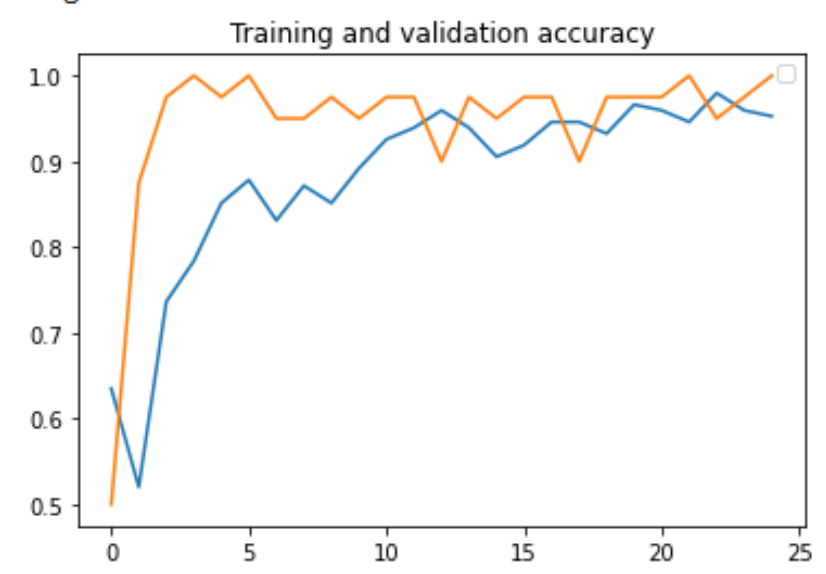

Fig. 2.Training and validation accuracy

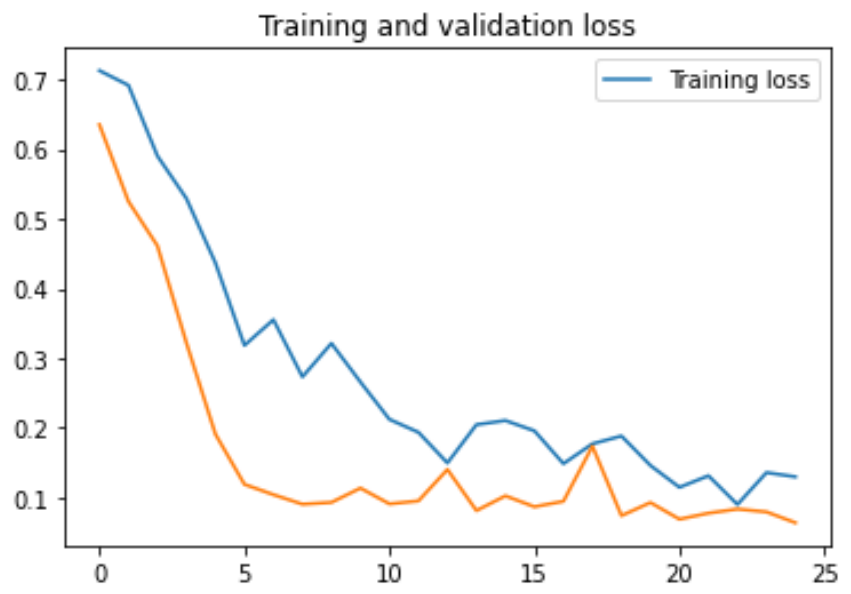

Fig.3.Training and validation loss.

Later on, the proposed framework's output can be used to examine clinically obtained Chest X rayimages with COVID19 disease. Furthermore, the proposed technique should be tested on a wider group of patients with COVID-19 positive Chest Xray and CT scan images. 


\section{References}

[1] Application of deep learning for fast detection of COVID-19 in X-Rays using nCOVnet, Harsh Panwar , P.K. Gupta , Mohammad Khubeb Siddiqui , Ruben Morales-Menendez , Vaishnavi Singh 2020, Chaos, Solitons and Fractals , vol.138,pp1-8

[2] Diagnosis and detection of infected tissue of COVID-19 patients based on lung x-ray image using convolutional neural network approaches, Shayan Hassantabar , Mohsen Ahmadi , Abbas Sharifi, 2020, Chaos, Solitons and Fractals, vol. 140

[3] Hybrid deep learning for detecting lung diseases from X-ray images, Subrato Bharati , Prajoy Podder, M. Rubaiyat Hossain Mondal , 2020, Informatics in Medicine Unlocked, vol.20

[4] COVID faster R-CNN: A novel framework to Diagnose Novel Coronavirus Disease (COVID-19) in X-Ray images, Kabid Hassan Shibly, Samrat Kumar Dey, Md Tahzib-Ul Islam, Md Mahbubur Rahman,2020, Informatics in Medicine Unlocked, vol. 20

[5] A combined deep CNN-LSTM network for the detection of novel coronavirus (COVID-19) using X-ray images, 2020, Informatics in Medicine Unlocked 20, 100412

[6] Deep Learning System to Screen Coronavirus Disease, Pneumonia,,Xiaowei Xu1 et al,2019.

[7] Online COVID-19 diagnosis with chest CT images:Lesion-attention deep neural networks, Bin Liu et al., MedRxiv, 2020

[8] Deep transfer learning-based automated detection of COVID-19 from lung CT scan slices, Sakshi Ahuja · Bijaya Ketan Panigrahi · Nilanjan Dey · Venkatesan Rajinikanth · Tapan Kumar Gandhi, Applied Intelligence, 2020. 